\title{
The Proposed Modified Liu System with Fractional Order
}

\author{
Alireza K. Golmankhaneh, ${ }^{1}$ Roohiyeh Arefi, ${ }^{1}$ and Dumitru Baleanu ${ }^{2,3,4}$ \\ ${ }^{1}$ Department of Physics, Urmia Branch, Islamic Azad University, P.O. Box 969, Oromiyeh, Iran \\ ${ }^{2}$ Department of Mathematics and Computer Science, Cankaya University, 06530 Ankara, Turkey \\ ${ }^{3}$ Institute of Space Sciences, P.O. Box MG-23, 76900 Magurele-Bucharest, Romania \\ ${ }^{4}$ Department of Chemical and Materials Engineering, Faculty of Engineering, King Abdulaziz University, \\ P.O. Box 80204, Jeddah 21589, Saudi Arabia
}

Correspondence should be addressed to Dumitru Baleanu; dumitru@cankaya.edu.tr

Received 27 February 2013; Accepted 15 March 2013

Academic Editor: J. A. Tenreiro Machado

Copyright (C) 2013 Alireza K. Golmankhaneh et al. This is an open access article distributed under the Creative Commons Attribution License, which permits unrestricted use, distribution, and reproduction in any medium, provided the original work is properly cited.

\begin{abstract}
The chaos in a new system with order 3 is studied. We have shown that this chaotic system again will be chaotic when the order of system is less than 3. Generalized Adams-Bashforth algorithm has been used for investigating in stability of fixed points and existence of chaos.
\end{abstract}

\section{Introduction}

It is well known that the nonlinear equations of dynamical systems with special condition have chaotic behavior [1]. Subsequently, additional studies were performed on the chaos and chaotic systems. Therefore solutions of different systems display their chaotic behavior such as Chen's system, Chua's dynamical system, the motion of double pendulum, and Rossler system amongst others. At first, it was thought that the chaos exists only when the order of system of differential equation is exactly 3 . When the system of differential equation is composed of three first order differential equations, the order of the system is the sum of orders. But later on, a very interesting thing was realized; that is, it is also possible to observe chaotic behavior in a fractional order system. The system is composed of differential equations with fractional order derivatives [1-28]. For example, Sheu et al. reviewed the chaotic behavior of the Newton-Leipnik system with fractional order [10]. The important thing in the study of fractional-order systems is the minimum effective dimension of the system for which the system remains chaotic. This quantity has been numerically calculated for different systems including fractional order Lorenz system [11], fractional order Chua's system [12], and fractional order Rössler system [13]. Recently, the chaos has been studied in fractional ordered Liu system, where the numerical investigations on the dynamics of this system have been carried out, and properties of the system have been analyzed by means of Lyapunov exponent [14]. In this paper we study the chaotic behavior of a generalization of the Liu system with fractional order.

The framework of the paper is as follows.

In Section 2, we study the behavior of a new fractional order system (modification of Liu system), and we study commensurate and incommensurate ordered systems and find lowest order at which chaos exists by numerical experiments. We have investigated the instability of fixed points and used Lyapunov exponent for the existence of chaos. In Section 3, we state the main conclusions.

\section{The Proposed Modified Liu System}

In this section, we review the condition for asymptotic stability of the commensurate and incommensurate fractional ordered systems. We suggest the readers to see [15-21] for the following section .

2.1. Asymptotic Stability of the Commensurate Fractional Ordered System. Let us consider the commensurate fractional ordered dynamical system equation

$$
D^{\alpha} x_{i}=f_{i}\left(x_{1}, x_{2}, x_{3}\right), \quad 1 \leq i \leq 3 .
$$


An equilibrium point of $(1)$ is $\mathbf{p} \equiv\left(x_{1}^{*}, x_{2}^{*}, x_{3}^{*}\right)$ which is $f_{i}(p)=0,1 \leq i \leq 3$, and a small disturbance from a fixed point is given by $\xi_{i}=x_{i}-x_{i}^{*}$. Thus, we have

$$
\begin{aligned}
D^{\alpha} \xi_{i}= & D^{\alpha} x_{i}=f_{i}\left(x_{1}, x_{2}, x_{3}\right)=f_{i}\left(\xi_{1}+x_{1}^{*}, \xi_{2}+x_{2}^{*}, \xi_{3}+x_{3}^{*}\right) \\
= & f_{i}\left(x_{1}^{*}, x_{2}^{*}, x_{3}^{*}\right)+\xi_{1} \frac{\partial f_{i}(p)}{\partial x_{1}}+\xi_{2} \frac{\partial f_{i}(p)}{\partial x_{2}} \\
& +\xi_{3} \frac{\partial f_{i}(p)}{\partial x_{3}}+\text { higher ordered terms } \\
\approx & \xi_{1} \frac{\partial f_{i}(p)}{\partial x_{1}}+\xi_{2} \frac{\partial f_{i}(p)}{\partial x_{2}}+\xi_{3} \frac{\partial f_{i}(p)}{\partial x_{3}} .
\end{aligned}
$$

Write the system in the matrix form

$$
D^{\alpha} \xi=J \xi
$$

where

$$
J=\left(\begin{array}{lll}
\partial_{1} f_{1}(p) & \partial_{2} f_{1}(p) & \partial_{3} f_{1}(p) \\
\partial_{1} f_{2}(p) & \partial_{2} f_{2}(p) & \partial_{3} f_{2}(p) \\
\partial_{1} f_{3}(p) & \partial_{2} f_{3}(p) & \partial_{3} f_{3}(p)
\end{array}\right)
$$

where $J$ is Jacobian matrix of the system, and if $J$ does not have purely imaginary eigenvalues, therefore the trajectories of the nonlinear system in the neighborhood of the equilibrium point have the same form as the trajectories of the linear system [18]. So we arrive at the following linear autonomous system:

$$
D^{\alpha} \xi=J \xi, \quad \xi(0)=\xi_{0},
$$

where $J$ is $n \times n$ matrix and $0<\alpha<1$. The system (5) is asymptotically stable if and only if $|\arg (\lambda)|>\alpha \pi / 2$ for all eigenvalues $\lambda$ of $J$. So for this condition the solutions $\xi_{i}(t)$ of (5) tend to 0 as $t \rightarrow \infty$. Therefore, the equilibrium point $p$ of the system is asymptotically stable if $|\arg (\lambda)|>\alpha \pi / 2$, for all eigenvalues $\lambda$ of $J$. For example,

$$
\min _{i}\left|\arg \left(\lambda_{i}\right)\right|>\frac{\alpha \pi}{2} .
$$

2.2. Asymptotic Stability of the Incommensurate Fractional Ordered System. Consider the following incommensurate fractional ordered dynamical system $[19,20]$. Now suppose the commensurate fractional ordered dynamical system equation

$$
D^{\alpha_{i}} x_{i}=f_{i}\left(x_{1}, x_{2}, x_{3}\right), \quad 1 \leq i \leq 3,
$$

where $0<\alpha_{i}<1$. One can write it as $\alpha_{i}=v_{i} / u_{i},\left(u_{i}, v_{i}\right)=1$, so $u_{i}, v_{i}$ are positive integers. The definition of common multiple of $u_{i}$ 's is $M$. Equilibrium point and small disturbance of the system $p$ and $\xi_{i}$ respectively as above. So we get

$$
D^{\alpha_{i}} x_{i} \approx \xi_{1} \frac{\partial f_{i}(p)}{\partial x_{1}}+\xi_{2} \frac{\partial f_{i}(p)}{\partial x_{2}}+\xi_{3} \frac{\partial f_{i}(p)}{\partial x_{3}}, \quad 1 \leq i \leq 3,
$$

where it can be written as

$$
\left(\begin{array}{l}
D^{\alpha_{1}} \xi_{1} \\
D^{\alpha_{2}} \xi_{2} \\
D^{\alpha_{3}} \xi_{3}
\end{array}\right)=J\left(\begin{array}{l}
\xi_{1} \\
\xi_{2} \\
\xi_{3}
\end{array}\right)
$$

where $J$ is Jacobian matrix evaluated at point $p$. The definition is

$$
\Delta(\lambda)=\operatorname{diag}\left(\left[\lambda^{M \alpha_{1}} \lambda^{M \alpha_{2}} \lambda^{M \alpha_{3}}\right]\right)-J,
$$

that is, if all the roots of equation $\Delta(\lambda)=0$ satisfy the condition $|\arg (\lambda)|>\pi / 2 M[21]$, the solution of linear system is asymptotically stable as follows:

$$
\frac{\pi}{2 M}-\min _{i}\left|\arg \left(\lambda_{i}\right)\right|<0
$$

The term in the left side of (11) is an instability measure for equilibrium point in fractional ordered system (IMFOS). Then, fractional order equation (7) exhibits chaotic attractor if the condition is $[19,20]$

$$
\text { IMFOS } \geq 0 .
$$

2.3. Modified Liu System. In this section we introduce the following system and show that the system is chaotic:

$$
\begin{aligned}
& \dot{x}=-a x-b y^{2}, \\
& \dot{y}=c y+d z x-e z^{2}, \\
& \dot{z}=f z+g x y,
\end{aligned}
$$

where $a=2, b=e=1, c=-3, d=-4, f=-7$, and $g=$ 4 with the initial conditions $(0.2,0,0.5)$ lead to the chaotic trajectories. Meanwhile, we want to show chaotic behavior of (13) involving fractional order. Also, we will calculate the minimum effective dimension by which the system remains chaotic. The corresponding fractional order system is

$$
\begin{aligned}
& D^{\alpha_{1}}=-a x-b y^{2}, \\
& D^{\alpha_{2}}=c y+d z x-e z^{2}, \\
& D^{\alpha_{3}}=f z+g x y,
\end{aligned}
$$

where $\alpha_{i} \in(0,1)$. In (14) if we choose $\alpha_{1}=\alpha_{2}=\alpha_{3}=$ $\alpha$, the system is called commensurate, and otherwise it is incommensurate. Now, we have four real equilibrium points for (13) which are shown in Table 1. In Table 1, we see the equilibrium points and the eigenvalues of the corresponding Jacobian matrix

$$
J=\left(\begin{array}{ccc}
-a & -2 b y & 0 \\
d z & c & d x-2 e z \\
g y x & g x & f
\end{array}\right)
$$

A saddle point $p$ is stable, if the Jacobian matrix has at least one eigenvalue with a negative real part. Otherwise, one eigenvalue with a nonnegative real part is called unstable. 
TABLE 1: Equilibrium points and corresponding eigenvalues.

\begin{tabular}{lcr}
\hline Equilibrium point & Eigenvalue & Nature \\
\hline$E_{0}(0,0,0)$ & $(-7,3,-2)$ & Saddle point \\
$E_{1}(-24.392,-6.98456,97.3529)$ & $(-64.4116,63.0432,-4.63163)$ & Saddle point \\
$E_{2}(-1.04307,1.44435,-0.860895)$ & $(-6.694,0.347 \pm 5.1057 i)$ & Saddle point \\
$E_{3}(-1.30636,-1.61639,1.20662)$ & $(-6.82593,0.412964 \pm 4.75973 i)$ & Saddle point \\
\hline
\end{tabular}

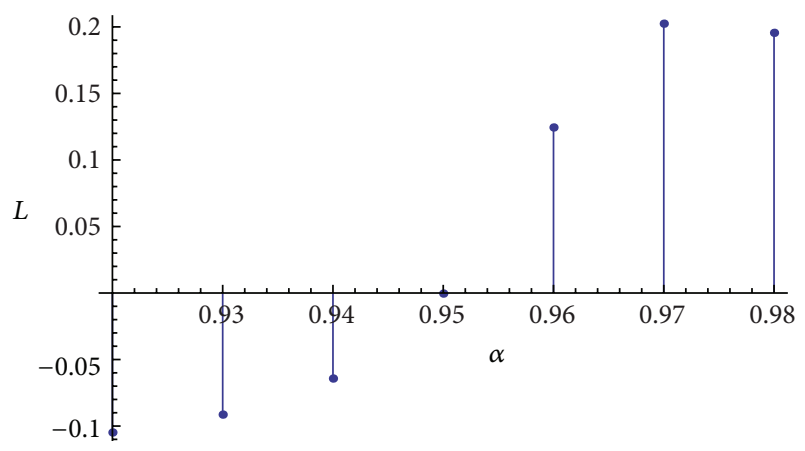

FIGURE 1: Lyapunov exponent.

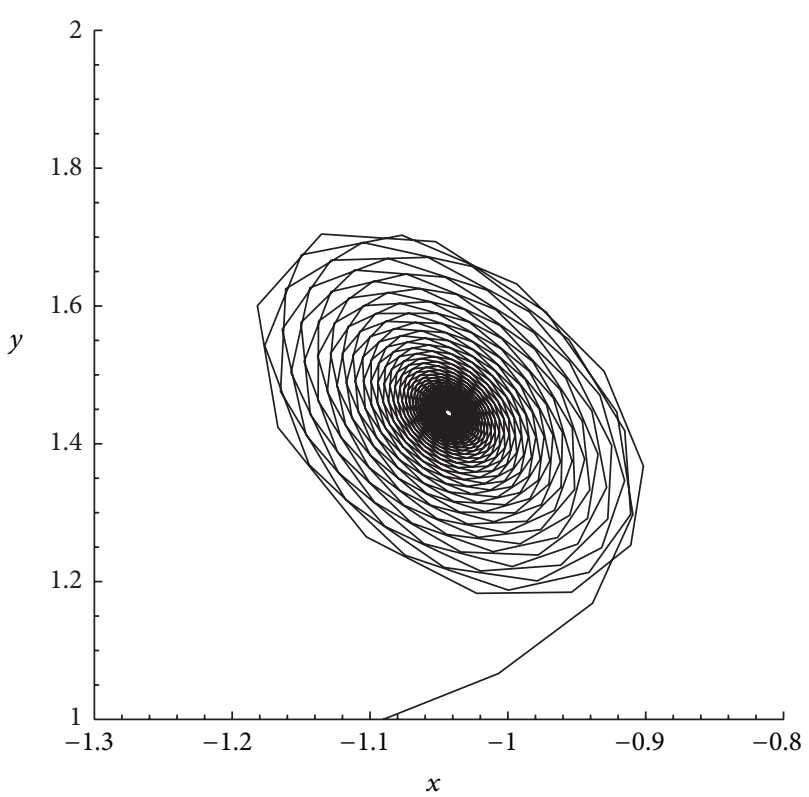

FIGURE 2: Phase portrait $\alpha=0.95$.

And saddle points have index one or two if there is exactly one or two unstable eigenvalues, respectively. It is established in the literature [22-26] that scrolls are generated only around the saddle points of an index one or two. Saddle points of index one are to connect scrolls. Table 1 shows that the equilibrium points $E_{1}$ and $E_{2}$ are saddle points of index two; therefor we have two-scroll attractor [22], in the fractional system given by (14).

2.4. Commensurate Ordered System. Consider the system equation (14), and let $\alpha_{1}=\alpha_{2}=\alpha_{3}=\alpha$, so it is called

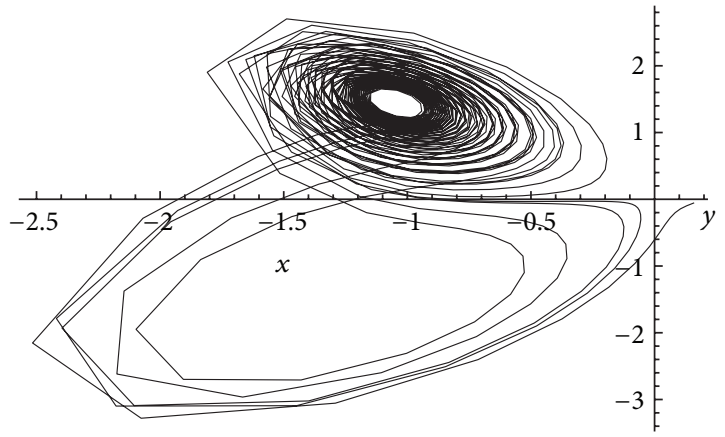

FIGURE 3: Phase portrait $x-y \quad \alpha=0.96$.

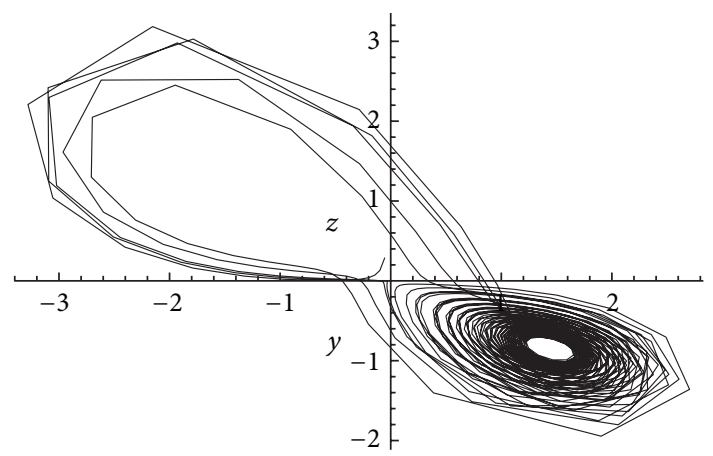

FIGURE 4: Phase portrait $y-z \alpha=0.96$.

commensurate order for this case. In this case a system shows regular behavior if it satisfies $\min _{i}\left|\arg \left(\lambda_{i}\right)\right|>\alpha \pi / 2$, then we have [15-21]

$$
\alpha<\frac{2}{\pi} \min _{i}\left|\arg \left(\lambda_{i}\right)\right| \approx 0.95
$$

From Figure 1 we can see that the Lyapunov exponent for the case of commensurate ordered equation (14) is positive if $\alpha>0.95$ [27, 28]. Thus we can realize that the system does not indicate chaotic behavior for the value $\alpha<0.95$. This corollary has been figured out by numerical outcome. Moreover, Figure 2 illustrates the phase portrait in $x y$-plane for the $\alpha=0.95$. Numerical experiments and Figures 3 and 4 demonstrate that the system has chaotic behavior for $\alpha=$ 0.96 . In addition, Figures 5 and 6 represent solutions $x(t)$ and $y(t)$ for $\alpha=0.96$, respectively. Adams-Bashforth predictorcorrector algorithm is used for numerical result with step size 0.1 . 


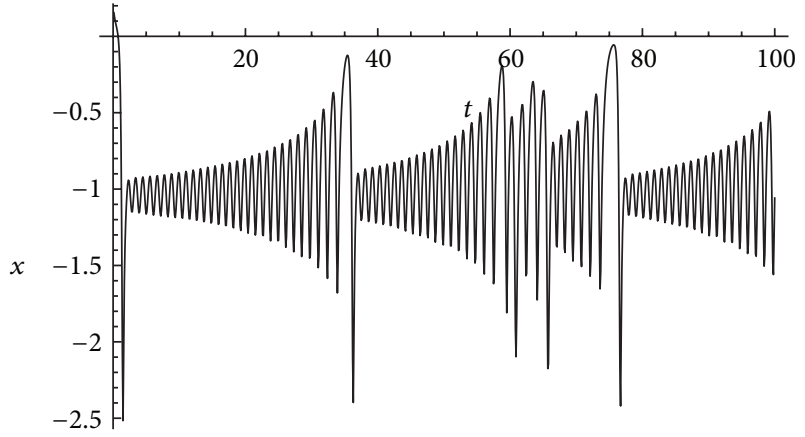

FIGURE 5: Solution $x(t)$ for $\alpha=0.96$.

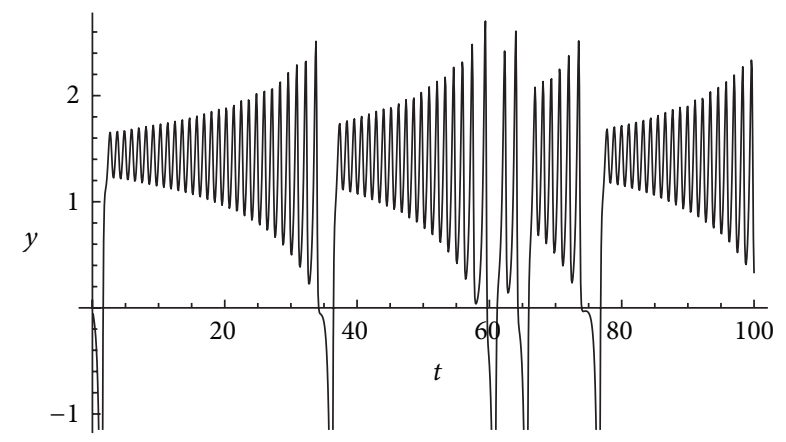

FiguRE 6: Solution $y(t)$ for $\alpha=0.96$.

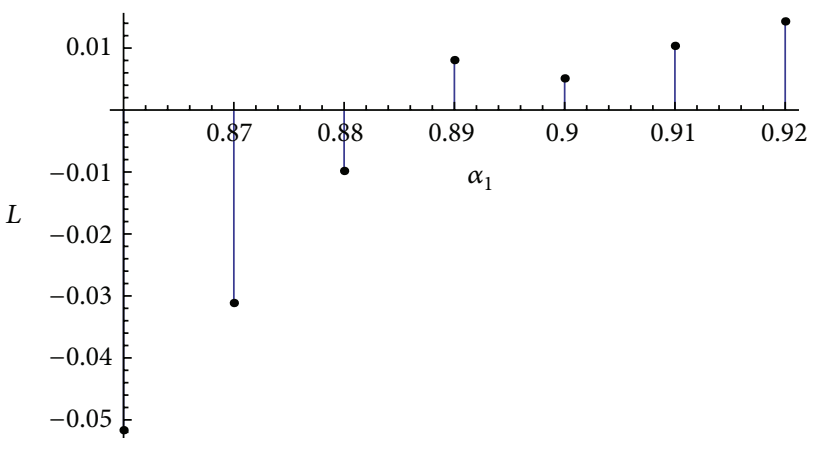

FIGURE 7: Lyapunov exponent- $\alpha_{1}$.

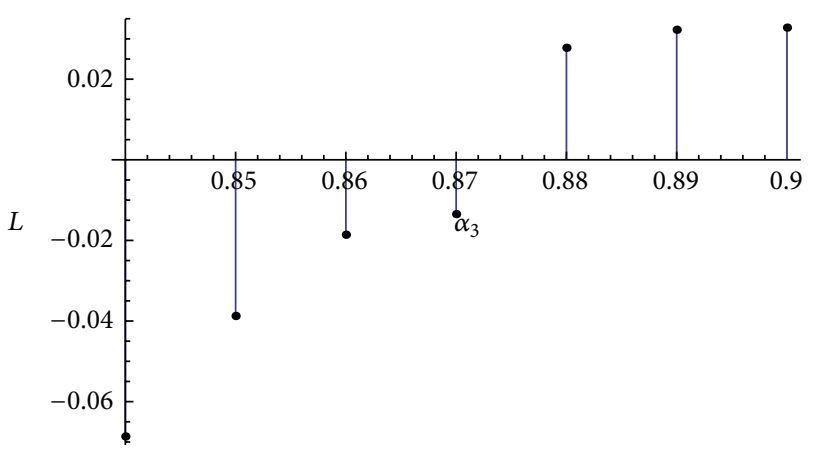

FIgURE 8: Lyapunov exponent- $\alpha_{3}$.

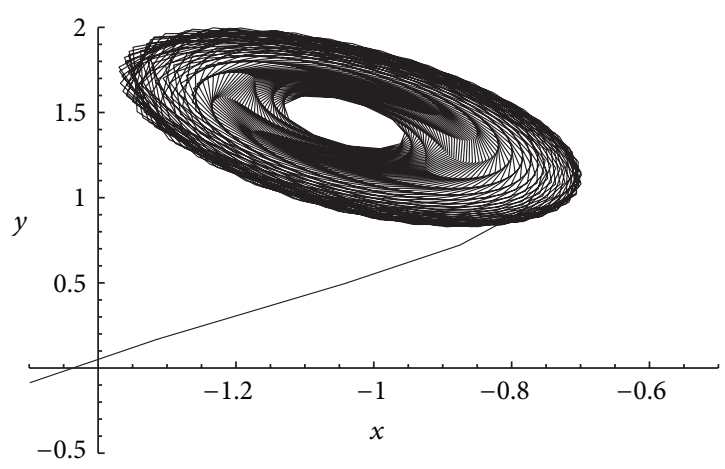

FIGURE 9: Phase portrait $x-y \alpha_{1}=0.88, \alpha_{2}=\alpha_{3}=1$.

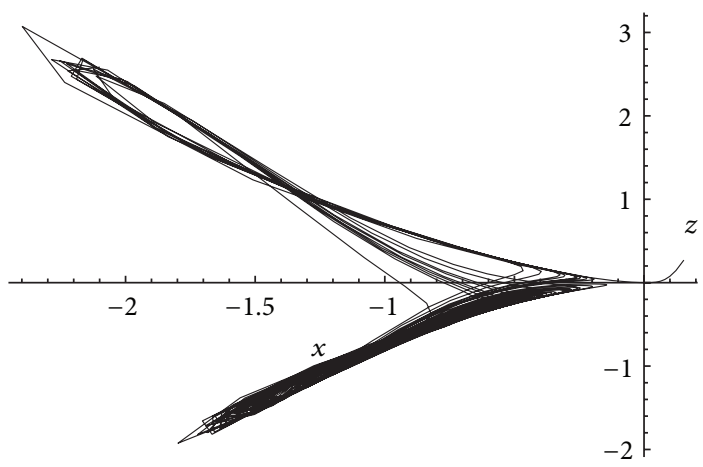

FIGURE 10: Phase portrait $x-z \alpha_{1}=0.89, \alpha_{2}=\alpha_{3}=1$.

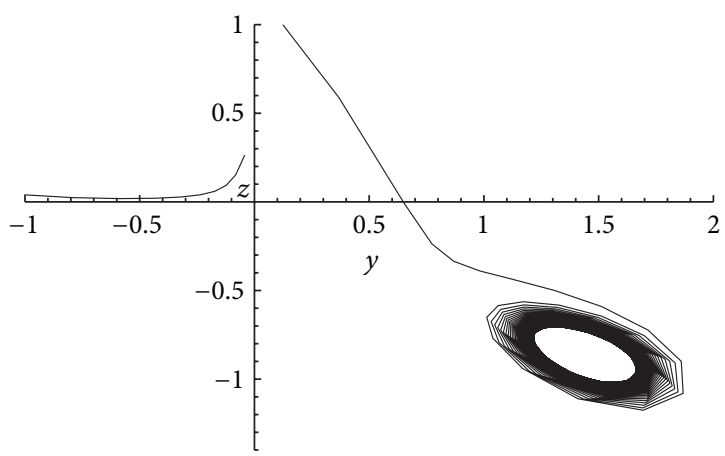

FIGURE 11: Phase portrait $y-z \alpha_{3}=0.87, \alpha_{2}=\alpha_{1}=1$.

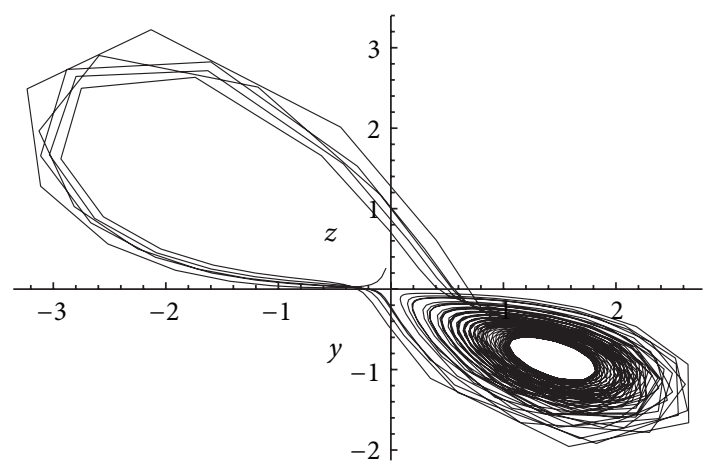

FIGURE 12: Phase portrait $y-z \alpha_{3}=0.88, \alpha_{2}=\alpha_{1}=1$. 
2.5. Incommensurate Ordered System. In this section we indicate that the condition for being chaotic system in the case of commensurate is not sufficient for the incommensurate case. So let us consider the fractional order system equation (14). Figures 7 and 8 display that the Lyapunov exponent is positive for $\alpha_{1} \geq 0.89, \alpha_{2}=\alpha_{3}=1$, and for the case $\alpha_{3} \geq 0.88, \alpha_{1}=\alpha_{3}=1$, respectively. Now, let us consider the following cases for (14).

(1) In the first condition we choose $\alpha_{1}=22 / 25, \alpha_{2}=\alpha_{3}=$ 1 . Therefore, one can acquire $M=\operatorname{LCM}(25,1,1)=25$. Since we have $\Delta(\lambda)=\operatorname{diag}\left(\left[\lambda^{22} \lambda^{25} \lambda^{25}\right]\right)-J\left(E_{1}\right)$, then

$$
\begin{aligned}
\operatorname{det}(\Delta(\lambda))= & -1.9 \times 10^{-4}-9.5 \times 106 \\
& -3 \lambda^{22}+5.45 \times 10^{-3} \lambda^{25} \\
& +4 \lambda^{47}+2 \lambda^{50}+\lambda^{72}
\end{aligned}
$$

And the instability measure for equilibrium point in fractional ordered system (IMFOS) will be for the system

$$
\frac{\pi}{50}-0.0=0.0628>0
$$

In Figure 9, there is no chaos, whereas IMFOS $>0$ [15-21]. This consequence leads to that the IMFOS $>0$ is not sufficient for existence of chaos.

(2) As second case, suppose $\alpha_{1}=89 / 100, \alpha_{2}=\alpha_{3}=1$, so that in this case the $M=\operatorname{LCM}(100,1,1)=100$. Also we have $\Delta(\lambda)=\operatorname{diag}\left(\left[\lambda^{89} \lambda^{100} \lambda^{100}\right]\right)-J\left(E_{1}\right)$, as well as we obtain

$$
\begin{aligned}
\operatorname{det}(\Delta(\lambda))= & -1.9 \times 10^{-4}-9.5 \times 10^{-3} \lambda^{89} \\
& +5.5 \times 10^{-3} \lambda^{100}+4 \lambda^{189} \\
& +2 \lambda^{200}+\lambda^{289} .
\end{aligned}
$$

Therefore, the IMFOS of the system for this state is

$$
\frac{\pi}{200}-0.0=0.0157>0 \text {. }
$$

If we look at Figure 10 we conclude the chaotic behavior for the system with the mentioned condition. Here we remark the lowest dimension of the system which has chaos (Figure 11).

(3) As third case, let $\alpha_{3}=43 / 50, \alpha_{1}=\alpha_{3}=1$. So with some manipulation we get $M=\operatorname{LCM}(1,1,50)=50$,

$$
\begin{aligned}
\operatorname{det}(\Delta(\lambda))= & -18807.7+5430.19 \lambda^{43} \\
& -9484.56 \lambda^{50}-\lambda^{93} \\
& +7 \lambda^{100}+\lambda^{143} .
\end{aligned}
$$

If we compute the IMFOS for the system, we will arrive at

$$
\frac{\pi}{100}-0.0=0.0314>0 \text {. }
$$

Figure 11 and (22) point that the system does not have chaotic condition.
(4) For the fourth case, we consider $\alpha_{3}=22 / 25, \alpha_{1}=$ $\alpha_{3}=1$. Therefore, we will obtain $M=\operatorname{LCM}(1,1,50)=50$,

$$
\begin{aligned}
\operatorname{det}(\Delta(\lambda))= & -1.9 \times 10^{-4} \\
& -9.5 \times 106-3 \lambda^{22} \\
& +5.45 \times 10^{-3} \lambda^{25}+4 \lambda^{47}+2 \lambda^{50}+\lambda^{72} .
\end{aligned}
$$

IMFOS for the fourth case is

$$
\frac{\pi}{100}-0.0=0.0628>0 \text {. }
$$

Numerical results and Figure 12 indicate for the this case that the system is chaotic.

\section{Conclusions}

A fractional order of a new system is investigated. Numerical calculations are performed for different values of fractional order. Lyapunov exponents and analytical conditions given in the literature have been used to check the existence of chaos. A minimum effective dimension is calculated for commensurate fractional order. Mathematica 7 has been used for computations in this paper.

\section{References}

[1] E. N. Lorenz, "Deterministic nonperiodic flow," Journal of The Atmospheric Sciences, vol. 20, pp. 130-141, 1963.

[2] J. Lü, T. Zhou, G. Chen, and S. Zhang, "Local bifurcations of the Chen system," International Journal of Bifurcation and Chaos, vol. 12, no. 10, pp. 2257-2270, 2002.

[3] L.J.M. Kocić, S. Gegovka-Zajkovka, and S. Kostadinova, "On Chua dynamical system," Applied Mathematics, Informatics \& Mechanics, Series A, vol. 2, pp. 53-60, 2010.

[4] T. Stachowiak and T. Okada, "A numerical analysis of chaos in the double pendulum," Chaos, Solitons and Fractals, vol. 29, no. 2, pp. 417-422, 2006.

[5] W. Xuedi and W. Chen, "Bifurcation analysis and control of the Rossler," in Proceedings of the 7th International Conference on System Natural Computation (ICNC '11), vol. 3, pp. 1484-1488, IEEE, Shanghai, China, 2011.

[6] K. S. Miller and B. Ross, An Introduction to the Fractional Calculus and Fractional Differential Equations, John Wiley \& Sons, New York, NY, USA, 1993.

[7] C. M. Ionescu and R. De Keyser, "Relations between fractionalorder model parameters and lung pathology in chronic obstructive pulmonary disease," IEEE Transactions on Biomedical Engineering, vol. 56, no. 4, pp. 978-987, 2009.

[8] J. A. T. Machado, "Entropy analysis of integer and fractional dynamical systems," Nonlinear Dynamics, vol. 62, no. 1-2, pp. 371-378, 2010.

[9] A. M. Lopes, J. A. T. Machado, C. M. A. Pinto, and A. M. S. F. Galhano, "Fractional dynamics and MDS visualization of earthquake phenomena," Computers and Mathematics with Applications, 2013.

[10] L.-J. Sheu, H.-K. Chen, J.-H. Chen et al., "Chaos in the Newton-Leipnik system with fractional order," Chaos, Solitons \& Fractals, vol. 36, no. 1, pp. 98-103, 2008. 
[11] I. Grigorenko and E. Grigorenko, "Chaotic dynamics of the fractional Lorenz system," Physical Review Letters, vol. 91, no. 3, pp. 034101/1-034101/4, 2003.

[12] T. T. Hartley, C. F. Lorenzo, and H. K. Qammer, "Chaos in a fractional order Chua's system," IEEE Transactions on Circuits and Systems I, vol. 42, no. 8, pp. 485-490, 1995.

[13] C. Li and G. Chen, "Chaos and hyperchaos in the fractionalorder Rössler equations," Physica A, vol. 341, no. 1-4, pp. 55-61, 2004.

[14] V. Daftardar-Gejji and S. Bhalekar, "Chaos in fractional ordered Liu system," Computers \& Mathematics with Applications, vol. 59, no. 3, pp. 1117-1127, 2010.

[15] I. Podlubny, Fractional Differential Equations, vol. 198 of Mathematics in Science and Engineering, Academic Press, San Diego, Calif, USA, 1999.

[16] S. G. Samko, A. A. Kilbas, and O. I. Marichev, Fractional Integrals and Derivatives, Gordon and Breach, New York, NY, USA, 1993.

[17] K. Diethelm, N. J. Ford, and A. D. Freed, "A predictor-corrector approach for the numerical solution of fractional differential equations," Nonlinear Dynamics, vol. 29, no. 1-4, pp. 3-22, 2002.

[18] Z. Vukic, Lj. Kuljaca, D. Donlagic, and S. Tesnjak, Non-linear Control Systems, Marcel Dekker, New York, NY, USA, 2003.

[19] A. Razminia, V.J. Majd, and D. Baleanu, "Chaotic incommensurate fractional order Rössler system: active control and synchronization," Advances in Difference Equations, article 15, 2011.

[20] M. S. Tavazoei and M. Haeri, "Chaotic attractors in incommensurate fractional order systems," Physica D, vol. 237, no. 20, pp. 2628-2637, 2008.

[21] W. Deng, C. Li, and J. Lü, "Stability analysis of linear fractional differential system with multiple time delays," Nonlinear Dynamics, vol. 48, no. 4, pp. 409-416, 2007.

[22] M. S. Tavazoei and M. Haeri, "A necessary condition for double scroll attractor existence in fractional-order systems," Physics Letters A, vol. 367, no. 1-2, pp. 102-113, 2007.

[23] L. O. Chua, M. Komuro, and T. Matsumoto, "The double scroll family. I. Rigorous proof of chaos," IEEE Transactions on Circuits and Systems, vol. 33, no. 11, pp. 1072-1097, 1986.

[24] C. P. Silva, "Shilnikov theorem-a tutorial," IEEE Transactions on Circuits and Systems I, vol. 40, no. 10, pp. 675-682, 1993.

[25] D. Cafagna and G. Grassi, "New 3D-scroll attractors in hyperchaotic Chua's circuits forming a ring," International Journal of Bifurcation and Chaos, vol. 13, no. 10, pp. 2889-2903, 2003.

[26] J. Lü, G. Chen, X. Yu, and H. Leung, "Design and analysis of multiscroll chaotic attractors from saturated function series," IEEE Transactions on Circuits and Systems I, vol. 51, no. 12, pp. 2476-2490, 2004.

[27] A. Wolf, J. B. Swift, H. L. Swinney, and J. A. Vastano, "Determining Lyapunov exponents from a time series," Physica D, vol. 16, no. 3, pp. 285-317, 1985.

[28] M. T. Rosenstein, J. J. Collins, and C. J. De Luca, "A practical method for calculating largest Lyapunov exponents from small data sets," Physica D, vol. 65, no. 1-2, pp. 117-134, 1993. 


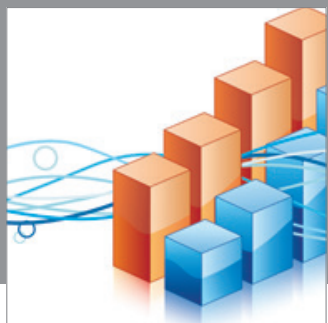

Advances in

Operations Research

mansans

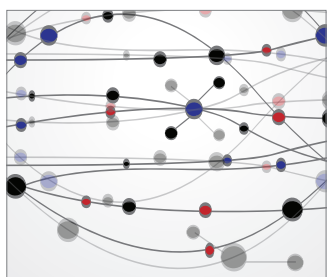

The Scientific World Journal
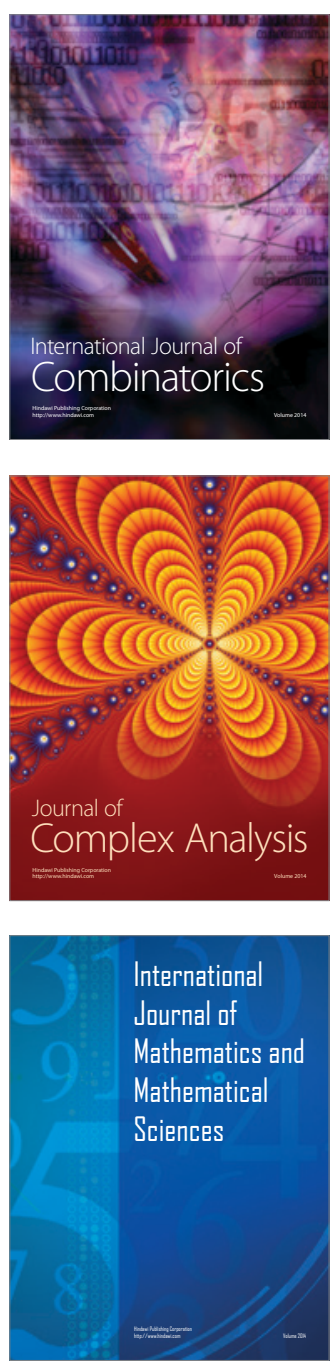
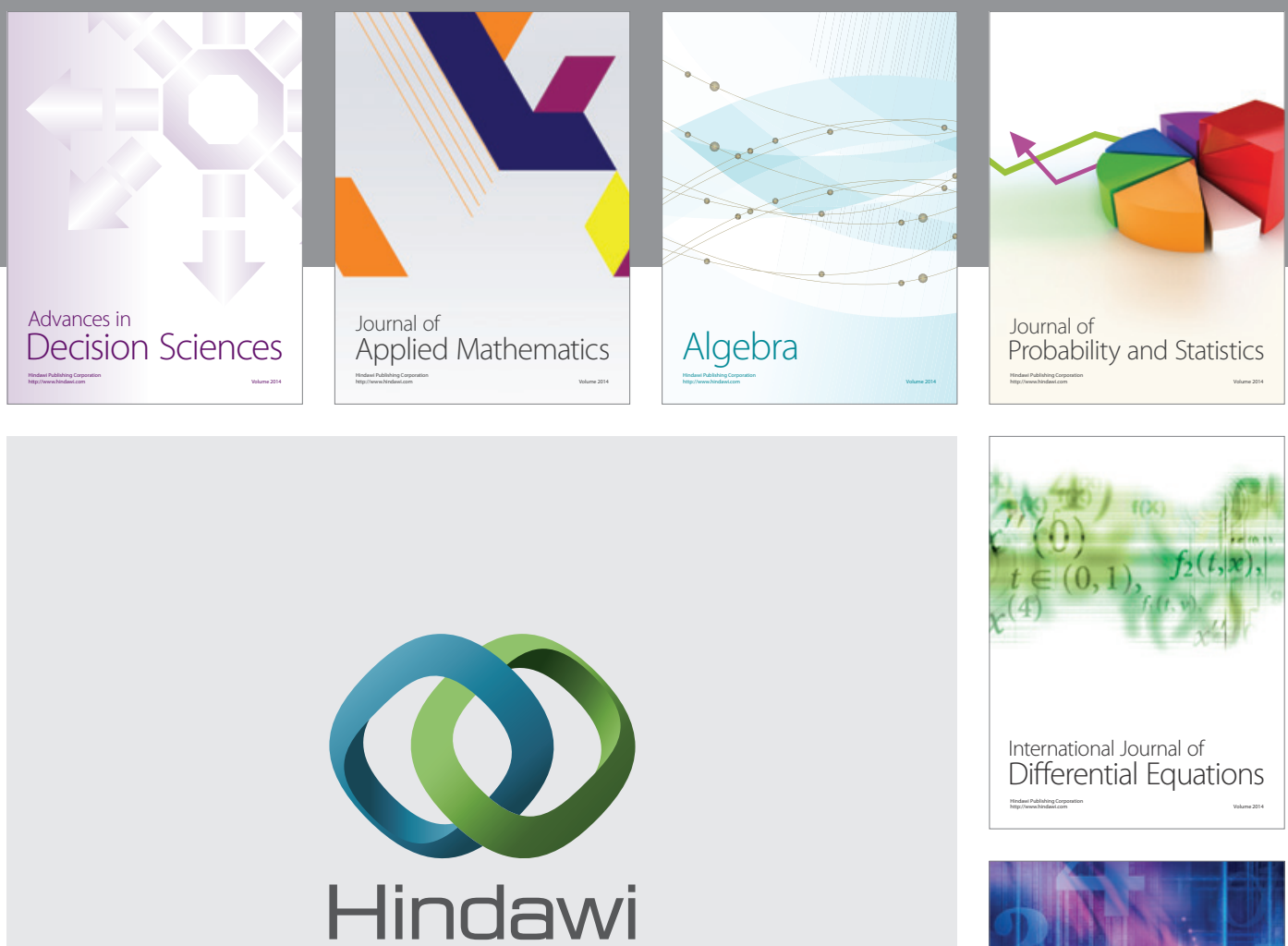

Submit your manuscripts at http://www.hindawi.com
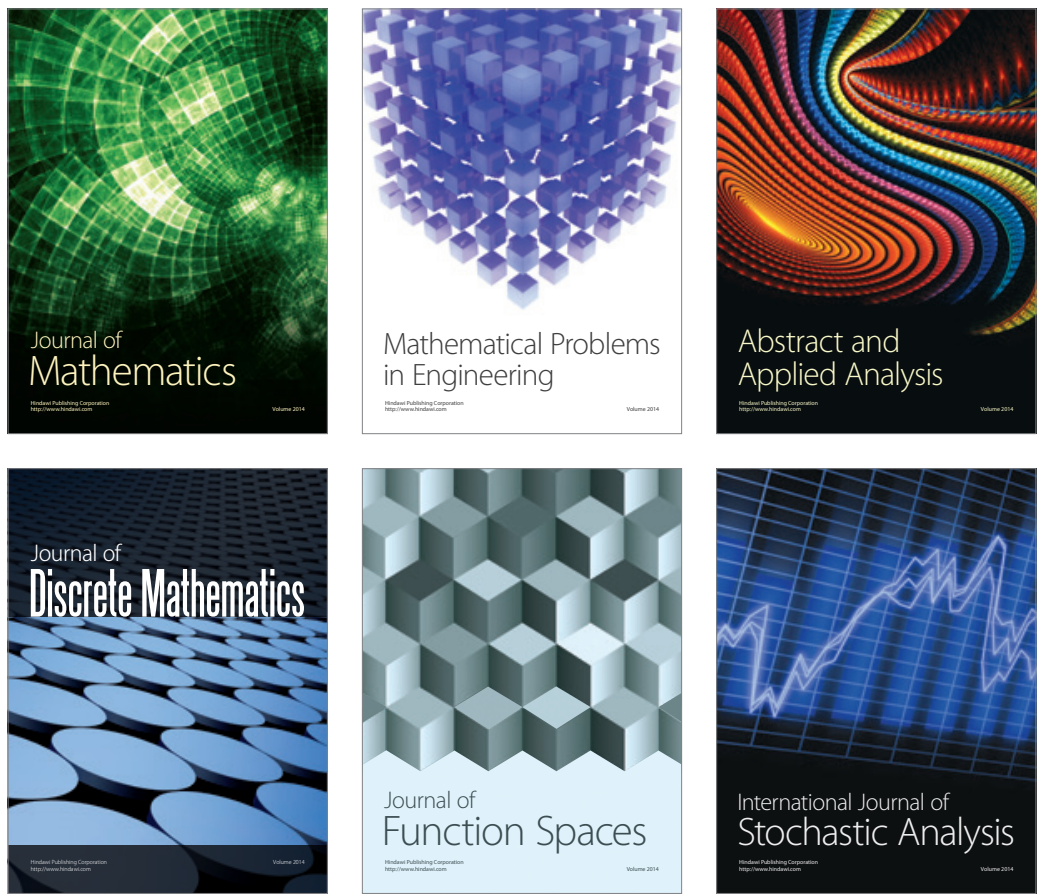

Journal of

Function Spaces

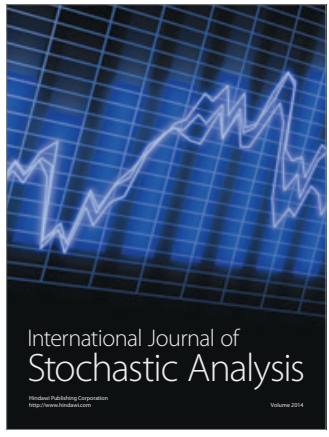

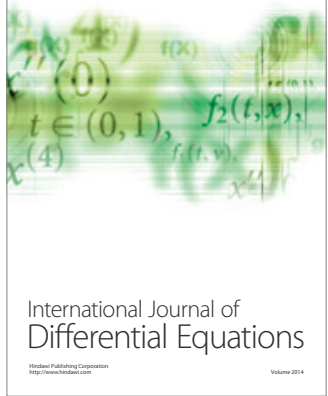
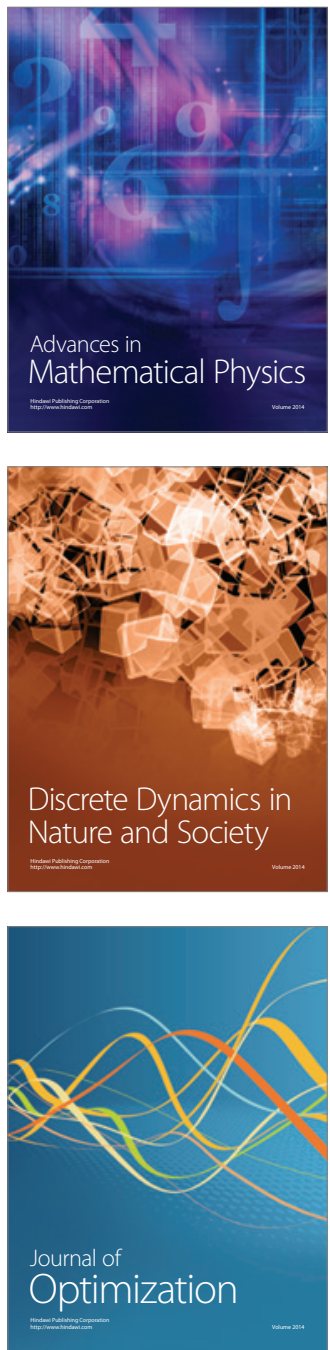